The Astrophysical Journal, 682:1103-1113, 2008 August 1

(C) 2008. The American Astronomical Society. All rights reserved. Printed in U.S.A.

\title{
SUBMILLIMETER OBSERVATIONS OF THE ISOLATED MASSIVE DENSE CLUMP IRAS 20126+4104
}

\author{
Hiroko Shinnaga, ${ }^{1}$ Thomas G. Phillips, ${ }^{1,2}$ Ray S. Furuya, ${ }^{3}$ and Riccardo Cesaroni ${ }^{4}$ \\ Received 2007 September 17; accepted 2008 April 10
}

\begin{abstract}
We imaged the 350 and $450 \mu \mathrm{m}$ continuum and $\mathrm{CO} J=6-5$ line emission of the IRAS $20126+4104$ clump at the CSO. The continuum and line observations show that the clump has a radius of $\sim 0.5 \mathrm{pc}$. The clump has a radial density profile $\propto r^{-1.2}$ for $r \lesssim 0.1 \mathrm{pc}$ and has $\propto r^{-2.3}$ for $r \gtrsim 0.1 \mathrm{pc}$, which suggests that the inner region is infalling, while the infall wave has not yet reached the outer region. Based on the $350 \mu \mathrm{m} / 450 \mu \mathrm{m}$ flux ratio map, three distinct regions are identified: a bipolar feature that coincides with the large scale CO bipolar outflow; a cocoon-like region that encases the bipolar feature and has a warm surface; and a cold layer outside of the cocoon region. The complex flux ratio pattern indicates that the clump is no longer uniform in terms of temperature as well as dust properties. The $\mathrm{CO}$ emission near the systemic velocity traces the dense clump and the outer layer of the clump shows narrow line widths $\left(\$ 3 \mathrm{~km} \mathrm{~s}^{-1}\right)$. The clump has a velocity gradient of $\sim 2 \mathrm{~km} \mathrm{~s}^{-1} \mathrm{pc}^{-1}$, which we interpret as due to rotation of the clump, as the equilibrium mass $\left(\sim 200 M_{\odot}\right)$ is comparable to the LTE mass. Over a scale of $\sim 1 \mathrm{pc}$, the clump rotates in the opposite sense with respect to the $\lesssim 0.03 \mathrm{pc}$ disk associated with the (proto)star. This is one of four objects in highmass and low-mass star-forming regions for which a discrepancy between the rotation sense of the envelope and the core has been found, suggesting that such a complex kinematics may not be unusual in star-forming regions.
\end{abstract}

Subject headings: infrared: ISM — ISM: clouds — ISM: jets and outflows — ISM: kinematics and dynamics — ISM: lines and bands — radio continuum: ISM — radio lines: ISM — stars: formation

\section{INTRODUCTION}

High-mass star formation, especially in its earliest phase, is poorly understood because of the nature of the objects in this phase. First of all, young high-mass stars are deeply embedded in their dense natal cloud cores and form and evolve on a short timescale, much shorter than that of low-mass star formation. Moreover, once a massive star has reached the zero-age main sequence, its strong UV radiation pressure heavily affects the surrounding material, thus making it difficult to trace the initial conditions of the molecular cloud. Finally, high-mass stars form in clusters at relatively large distances from us (typically a few $\mathrm{kpc}$ ); it is hence not easy to resolve individual young stellar objects, even for the closest massive star-forming region, Orion (e.g., Plambeck et al. 1982; Greenhill et al. 1998; Beuther et al. 2005).

The early B-type massive (proto)star, IRAS $20126+4104$, is a well-studied object which provides us the opportunity to study the early phase of massive star formation in a relatively simple configuration. This consists of a luminous IRAS source embedded in a dense core and associated with both a bipolar outflow and a circumstellar Keplerian disk (imaged in $\mathrm{NH}_{3}, \mathrm{CH}_{3} \mathrm{CN}$, and $\mathrm{C}^{34} \mathrm{~S}$; Zhang et al. 1998; Cesaroni et al. 1997, 1999, 2005). The Caltech Millimeter Array (OVRO-MMA) was used to image the large-scale $(\sim 0.5 \mathrm{pc})$ north-south outflow in detail using the $\mathrm{CO}$ $J=1-0$ transitions (Shepherd et al. 2000). It appears that this north-south direction is not preserved on a smaller scale $(0.1 \mathrm{pc})$ where a jet oriented southeast-northwest has been imaged in a variety of tracers, such as shocked $\mathrm{H}_{2}$ (Cesaroni et al. 1997;

\footnotetext{
1 California Institute of Technology Submillimeter Observatory (CSO), 111 Nowelo Street, Hilo, HI 96720; shinnaga@submm.caltech.edu.

2 Division of Physics, Mathematics, and Astronomy, California Institute of Technology, 1200 East California, Pasadena, CA 91125.

3 Subaru Telescope, National Astronomical Observatory of Japan, 650 North A’ohoku Place, Hilo, HI 96720.

${ }^{4}$ Osservatorio Astrofisico di Arcetri, INAF, Largo E. Fermi 5, 50125 Firenze,
}

Shepherd et al. 2000), $\mathrm{NH}_{3}(3,3) /(4,4)$ emission (Zhang et al. 1999), $\mathrm{SiO}$ and $\mathrm{HCO}^{+}$emission (Cesaroni et al. 1997, 1999), and scattered light in the near infrared (NIR) continuum (e.g., Ayala et al. 1998; Sridharan et al. 2005). The position angle (P.A.) of the jet is $\sim 150^{\circ}$, significantly different from that of the largescale molecular outflow (P.A. $\simeq-25^{\circ}$; Shepherd et al. 2000; Lebrón et al. 2006). Such a discrepancy has been interpreted as precession of the jet/outflow axis (Shepherd et al. 2000; Cesaroni et al. 2005).

While many studies have been done on this object, only a few of these have focused on the dense parsec-scale clump hosting the (proto)star. It is thus of interest to investigate the physical properties and evolution of such a clump and its interaction with the outflow from the (proto)star.

Here we present a study of the dense clump and bipolar outflow based on submillimeter continuum and spectroscopic observations taken with the $10.4 \mathrm{~m}$ Leighton telescope at the Caltech Submillimeter Observatory (CSO). ${ }^{5}$ Note that $1.5 \mathrm{kpc}$ is used as the distance of the object to estimate physical parameters in this paper. A discussion regarding the distance of this object is given in the Appendix.

\section{OBSERVATIONS AND DATA REDUCTION}

Submillimeter continuum and molecular line observations were carried out toward the massive dense clump (Table 1). The dust continuum observations at 350 and $450 \mu \mathrm{m}$ were made on 2005 May 10 and 11 using the Submillimeter High Angular Resolution Camera II (SHARC II; Dowell et al. 2003; Voellmer et al. 2003) mounted on a Nasmyth focus. Both the 350 and $450 \mu \mathrm{m}$ beams have simple Gaussian shapes with half-power beam widths $\left(\theta_{\text {HPBW }}\right)$ of $8.7^{\prime \prime}$ and $9.8^{\prime \prime}$ (see Table 1). The 384 pixels of the bolometer array cover a region of about $1^{\prime} \times 2.6^{\prime}$ at $350 \mu \mathrm{m}$. Pointing and focus were checked regularly. The pointing drift

\footnotetext{
5 The Caltech Submillimeter Observatory is supported by NSF grant AST-0229008.
} 
TABLE 1

OBSERVATIONS

\begin{tabular}{|c|c|c|c|c|c|c|c|}
\hline Emission & $\begin{array}{l}\text { Frequency } \\
\quad(\mathrm{GHz})\end{array}$ & $\begin{array}{l}\text { Bandwidth } \\
\text { (MHz) }\end{array}$ & Telescope/Instrument & $\begin{array}{l}\theta_{\mathrm{HPBW}}{ }^{\mathrm{a}} \\
(\operatorname{arcsec})\end{array}$ & $\begin{array}{c}\mathrm{FOV}^{\mathrm{b}} \\
\left(\operatorname{arcmin}^{2}\right)\end{array}$ & $\begin{array}{l}\text { Velocity Resolution } \\
\qquad\left(\mathrm{km} \mathrm{s}^{-1}\right)\end{array}$ & Reference \\
\hline $350 \mu \mathrm{m}$ continuum .................. & 849.4 & 102900 & CSO/SHARC II & 8.7 & $9 \times 9$ & $\ldots$ & This work \\
\hline $\mathrm{CO} J=6-5$ & 691.473076 & 50 & $\mathrm{CSO} / 690 \mathrm{GHz} \mathrm{Rx}$ & 10 & $2.7 \times 2.7$ & 0.6 & This work \\
\hline $\mathrm{CO} J=7-6 \ldots \ldots \ldots \ldots \ldots$ & 806.651776 & 250 & $\mathrm{HHT}^{\mathrm{c}} / 800 \mathrm{GHz} \mathrm{Rx}$ & 10 & $\ldots$ & 0.9 & Kawamura et al. (1999) \\
\hline
\end{tabular}

${ }^{\text {a }}$ Half-power beam width.

${ }^{\mathrm{b}}$ Field of view.

${ }^{c}$ The Heinrich Hertz Telescope.

due to temperature (Shinnaga 2004) was subtracted in the data reduction process. The final pointing accuracy is within $\sim 2^{\prime \prime}$. The Dish Surface Optimization System (DSOS; Leong 2005) was used during the observations; this allows us to apply a gravitational deformation correction to the main mirror physically and is thus a key component for high-sensitivity short submillimeter wave observations. Zenith opacities $\tau_{225} \sim 0.06$ at the wavelength of $1300 \mu \mathrm{m}$ were measured with the CSO tau meter during the $350 \mu \mathrm{m}$ observations, while during the acquisition of the $450 \mu \mathrm{m}$ data we measured $\tau_{225} \sim 0.09$. The data were reduced with the software Comprehensive Reduction Utility (CRUSH; Kovacs 2006) version 1.41. The flux was calibrated with Neptune for both wavelengths, with an estimated calibration uncertainty of $7 \%$ at $350 \mu \mathrm{m}$ and $5 \%$ at $450 \mu \mathrm{m}$. The estimated flux of the planet was $94.6 \mathrm{Jy}$ at $350 \mu \mathrm{m}$ on 2005 May 10 and $55.9 \mathrm{Jy}$ at $450 \mu \mathrm{m}$ on 2005 May 11. Note that the angular size of the planet was much smaller than the beam sizes at these wavelengths. An area of $\sim 9^{\prime}$ centered at the source position was mapped with the instrument, in box-scan mode. The rms noise of the final maps is about $200 \mathrm{mJy}^{\text {beam }}{ }^{-1}$ for both bands. After calibration, data analysis was done using the program GRAPHIC from the GILDAS software.

Spectroscopic observations in the CO $J=6-5$ line $(\nu=$ 691.473076 GHz; Goldsmith et al. 1981) were made on 2004 July 8 and 9 using a $690 \mathrm{GHz}$ heterodyne receiver. An area of $2.7^{\prime} \times 2.7^{\prime}$ was sampled with steps of $5^{\prime \prime}$ and $20^{\prime \prime}$, while the innermost region of $1^{\prime} 20^{\prime \prime} \times 1^{\prime}$ was sampled with steps of $5^{\prime \prime} . \tau_{225}$ was between 0.07 and 0.085 during the observations. The SIS receiver operation at $4 \mathrm{~K}$ produced typical single-sideband system temperatures (measured with the $1.5 \mathrm{GHz}$ bandwidth acoustooptic spectrometer) between $\sim 7000$ and $10,900 \mathrm{~K}$ at $691 \mathrm{GHz}$, for elevations in the range of $\sim 50^{\circ}-70^{\circ}$. The antenna temperature $T_{\mathrm{A}}^{*}$ calibration was done by the standard chopper wheel method every 30 minutes to $1 \mathrm{hr}$. The data were converted to the main-beam brightness temperature $\left(T_{\mathrm{mb}}\right)$ scale. The $\theta_{\mathrm{HPBW}}$ at $691 \mathrm{GHz}$ is $\sim 10^{\prime \prime}$. The main-beam efficiency at the frequency of the $\mathrm{CO} 6-5$ transition at an elevation of $49^{\circ}$ was estimated to be $\sim 40 \%$, through observations of Uranus. Pointing was checked approximately every $2 \mathrm{hr}$. The pointing accuracy is estimated to be $\sim 5^{\prime \prime}$. Daily gain variations as well as gain curve over different

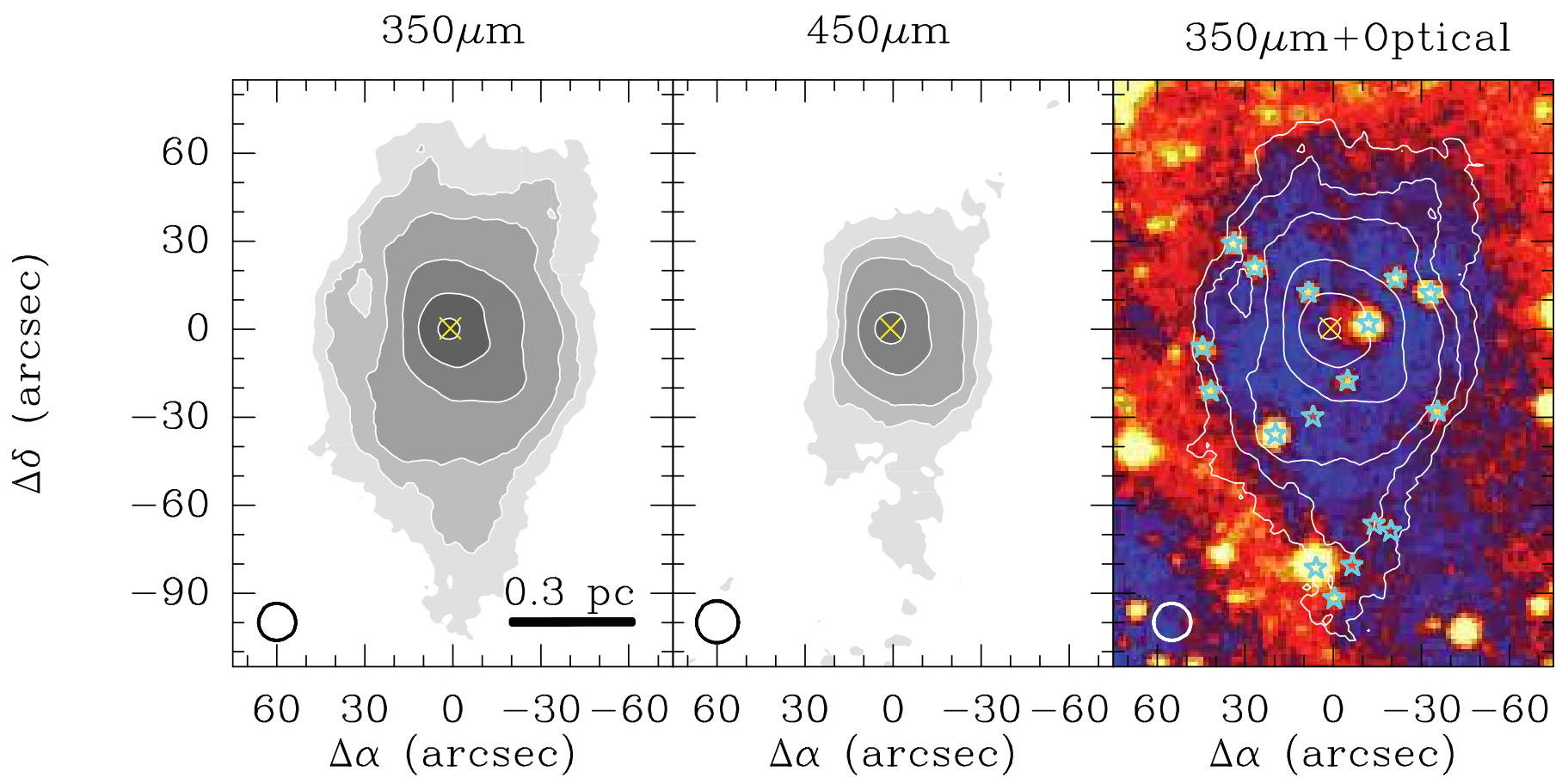

FIG. 1.-SHARC II $350 \mu \mathrm{m}$ (left) and $450 \mu \mathrm{m}$ (middle) images of the dense clump. The $350 \mu \mathrm{m}$ image is overlaid on the optical image (DSS $0.5 \mu \mathrm{m} ;$ colored shading) in the right panel. Blue stars mark the positions of foreground stars. Gray-scale contours of two images from the left are drawn at 2,5,9,27,81, and 243 $\sigma$, where $1 \sigma=$ $200 \mathrm{mJy} \mathrm{beam}^{-1}$. The yellow cross at the center represents the position of the clump center, namely the peak of the $350 \mu \mathrm{m}$ continuum emission. The beam is shown in the left bottom corner of each panel. 
TABLE 2

Peak Flux Densities and Total Flux

\begin{tabular}{|c|c|c|c|c|c|c|}
\hline $\begin{array}{l}\text { Wavelengths } \\
\qquad(\mu \mathrm{m})\end{array}$ & Telescope/Instrument & $\begin{array}{l}\theta_{\mathrm{HPBW}}{ }^{\mathrm{a}} \\
(\operatorname{arcsec})\end{array}$ & $\begin{array}{l}\text { Flux Density } \\
\left(\text { Jy beam }^{-1}\right)\end{array}$ & $\begin{array}{l}\text { Total Flux } \\
\text { (Jy) }\end{array}$ & $\begin{array}{l}\text { Effective Radius } \\
\quad(\operatorname{arcsec})\end{array}$ & Reference \\
\hline $350 \ldots \ldots \ldots \ldots$ & CSO/SHARC II & 8.7 & $59.1(4.1)$ & 477 (13) & 65.5 & This work \\
\hline 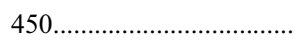 & CSO/SHARC II & 9.8 & $27.4(1.2)$ & $137(14)$ & 50.4 & This work \\
\hline $450 \ldots \ldots \ldots \ldots$ & JCMT/SCUBA & 8 & $29(0.9)$ & $91.1(3.9)$ & $\ldots$ & Williams et al. (2004) \\
\hline 450 & JCMT/SCUBA & 12 & $32.0(6.0)$ & $162(24)$ & $\ldots$ & Cesaroni et al. (1999) \\
\hline $1200 \ldots \ldots \ldots \ldots \ldots \ldots$ & Pico Veleta/MAMBO & 11 & $1.1(0.3)$ & 5.8 & 20 & Beuther et al. (2002) \\
\hline $1350 \ldots \ldots \ldots \ldots \ldots \ldots$ & JCMT/SCUBA & 22 & $1.74(0.20)$ & $\ldots$ & $\ldots$ & Cesaroni et al. (1999) \\
\hline 2000 & JCMT/SCUBA & 33 & $0.56(0.05)$ & $\ldots$ & $\ldots$ & Cesaroni et al. (1999) \\
\hline
\end{tabular}

${ }^{a}$ Half-power beam width.

${ }^{\mathrm{b}}$ Error is given in the parentheses when the literature provides.

elevation angles were derived and corrected using the $\mathrm{CO} 6-5$ spectrum of NGC 7027. Frequency calibration was done every 30 minutes. The data were reduced using the program CLASS of the GILDAS software.

\section{RESULTS AND ANALYSIS}

\subsection{Submillimeter Dust Continuum}

\subsubsection{Overall Structures and Flux Measurements}

Submillimeter continuum observations over a 4 pc region confirmed that the object is an isolated massive dense clump (Fig. 1). The submillimeter dust peak positions in both bands coincide with the 1 and $3 \mathrm{~mm}$ continuum peak position (R.A. = $20^{\mathrm{h}} 14^{\mathrm{m}} 26.03^{\mathrm{s}}$, Decl. $=41^{\circ} 13^{\prime} 32.7^{\prime \prime}[\mathrm{J} 2000.0] ; l=78^{\circ} 07^{\prime} 20.2^{\prime \prime}$, $\left.b=03^{\circ} 37^{\prime} 58.3^{\prime \prime}\right)$, measured by Cesaroni et al. (1999) and Shepherd et al. (2000).

The $350 \mu \mathrm{m}$ image traces a north-south elongated single dense clump with a size of $\sim 1.3 \times 0.7 \mathrm{pc}$, which fits the silhouette against diffuse ionized emission. The $450 \mu \mathrm{m}$ dust continuum, which has a size of about $0.9 \times 0.4 \mathrm{pc}$, looks more compact, with some extension toward the south. The clump has a prolate spheroidal shape. The effective radius of the clump is defined as $R_{\text {eff }}=$ $\left(A / \pi-\theta_{\mathrm{HPBW}}^{2} / 4\right)^{1 / 2}$, where $A$ is the area of $2 \sigma$ contour traced by 350 and $450 \mu \mathrm{m}$ continuum. We find $R_{\text {eff } 350}=0.48 \mathrm{pc}$ and $R_{\text {eff } 450}=0.37 \mathrm{pc}$, corresponding to angular radii of $66^{\prime \prime}$ and $51^{\prime \prime}$, respectively.

The peak flux values at 350 and $450 \mu \mathrm{m}$ are $59.1 \pm 4.1 \mathrm{Jy}$ beam $^{-1}$ and $27.4 \pm 1.2 \mathrm{Jy}$ beam $^{-1}$, respectively. Within the uncertainties, these values are consistent with previous flux measurements done with SCUBA (Cesaroni et al. 1999), with $\theta_{\mathrm{HPBW}}=$ $15^{\prime \prime}$, but the $350 \mu \mathrm{m}$ peak flux value is higher than another SHARC measurement at the same wavelength, $34 \mathrm{Jy}$ beam $^{-1}$ (Hunter et al. 2000 ), with $\theta_{\text {HPBW }}=11^{\prime \prime}$. All peak flux densities at different wavelengths reported to date are summarized in Table 2. Our total flux densities computed inside the $2 \sigma$ contour are $477 \pm 13 \mathrm{Jy}$ at $350 \mu \mathrm{m}$ and $137 \pm 14 \mathrm{Jy}$ at $450 \mu \mathrm{m}$, respectively.

\subsubsection{Radial Density Profile}

We approximate the column density $\left[N\left(\mathrm{H}_{2}\right)\right]$ as a function of clump radius $(r)$ as a power law, $N\left(\mathrm{H}_{2}\right) \propto r^{p}$. This reflects the density structure of the clump, which to a first-order approximation should also scale as a power law, with index $p-1$. Figure 2 represents the azimuthally averaged column density profile derived from the $350 \mu \mathrm{m}$ data. The density profile is plotted from the clump center, i.e., the peak position at $350 \mu \mathrm{m}$. A discussion on the effect of a temperature gradient on the estimate of the column density profile will be given in $\S 4.1$.

One can see that the inner part of the dense clump has a flatter index compared to that of outer part. The power law for $0.03 \lesssim$ $r \lesssim 0.13$ pc (corresponding to $4.4^{\prime \prime}$ and $17^{\prime \prime}$ ) is $r^{-0.26 \pm 0.03}$. Note that this region is larger than $\theta_{\mathrm{HPBW} 350} / 2=4.3^{\prime \prime}$, so that the flatter index observed in the inner region is not due to beam dilution. In the range $0.13 \lesssim r \lesssim 0.3 \mathrm{pc}$ (i.e., between $17^{\prime \prime}$ and $40^{\prime \prime}$ ), the column density decreases as $r^{-1.32 \pm 0.02}$. Finally, for $0.3 \lesssim r \lesssim 0.7 \mathrm{pc}$ $\left(40^{\prime \prime}-100^{\prime \prime}\right)$, the index $p$ is $-1.98 \pm 0.02$. Steepening of the index in this region is likely due to geometrical effects close to the clump border at $\sim 50^{\prime \prime}$ from the center. Instead, in the two inner regions the estimates obtained are reliable and imply power-law indices of -1.26 and -2.32 for the volume density. These issues will be further discussed in $\S 4.1$.

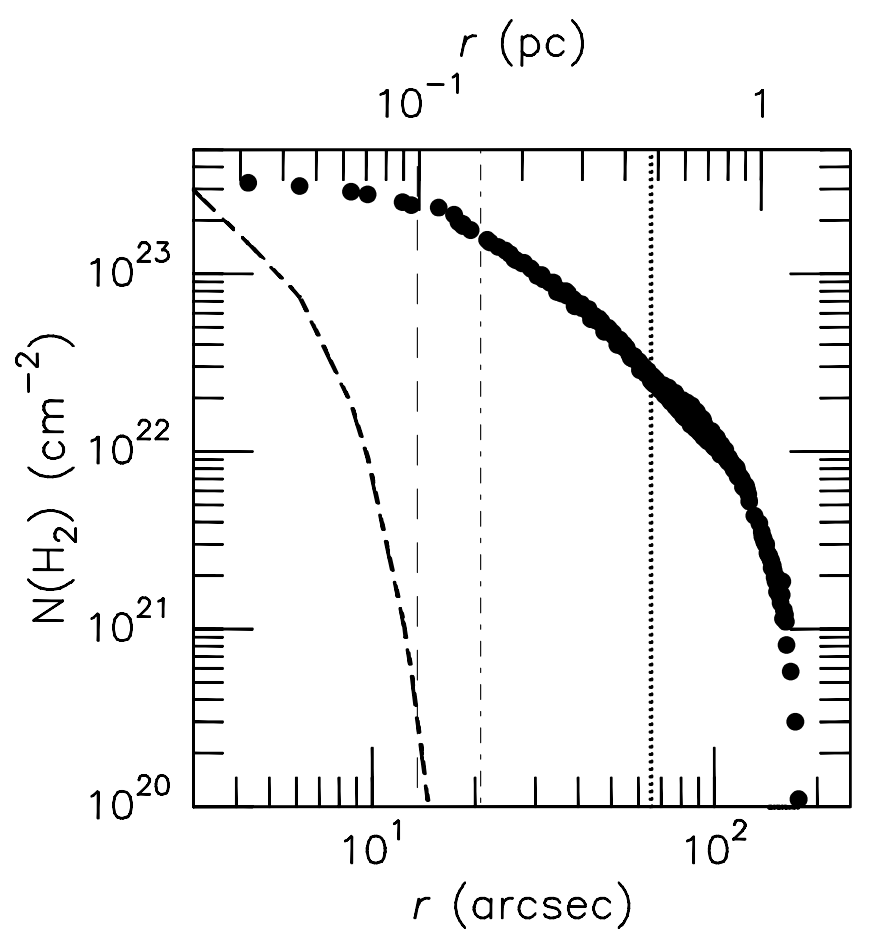

FIG. 2.-Radial column density profile of the dense clump (black dots) derived using the $350 \mu \mathrm{m}$ data. The thick broken line represents the beam shape. The vertical dotted line marks $R_{\text {eff } 350}$. The dash-dotted and long-dashed lines mark the effective radii of the continuum emission measured at $1.2 \mathrm{~mm}$ and at $850 \mu \mathrm{m}$, respectively. 


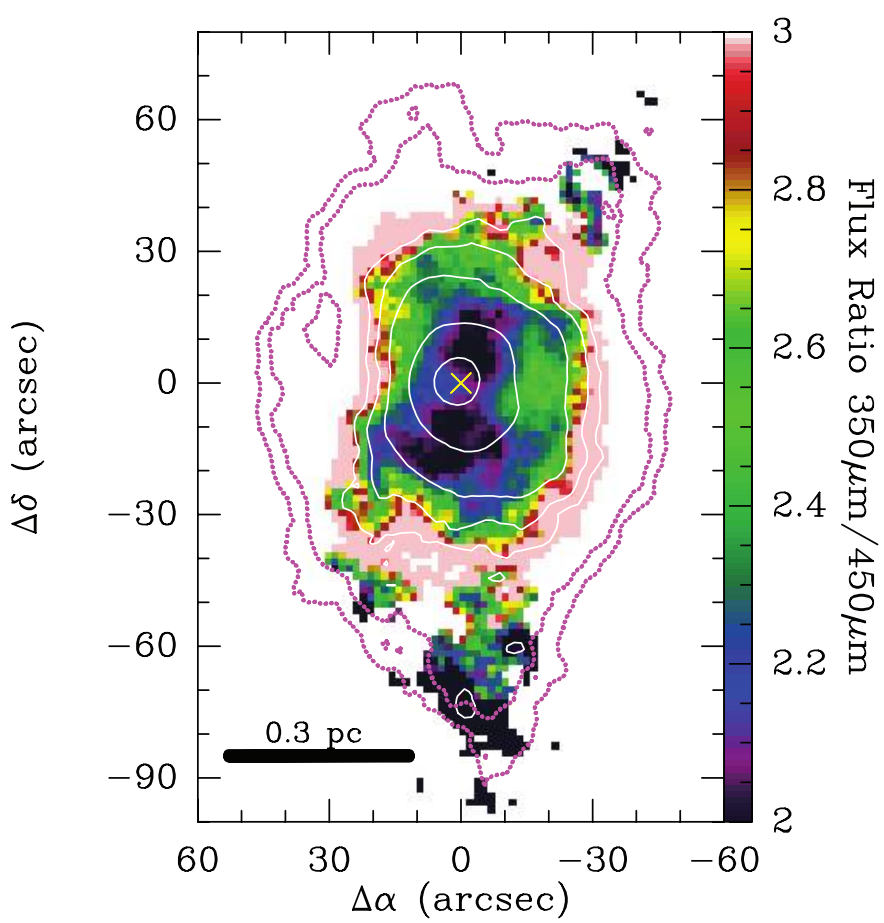

FIG. 3.-The $350 \mu \mathrm{m} / 450 \mu \mathrm{m}$ flux ratio map. The $450 \mu \mathrm{m}$ emission is drawn in white contours corresponding to $3,5,9,27$, and $81 \sigma$. The pink dotted contours correspond to the $3 \sigma$ and $5 \sigma$ levels of the $350 \mu \mathrm{m}$ emission. The yellow cross marks the clump center.

\subsection{3. $350 \mu \mathrm{m} / 450 \mu \mathrm{m}$ Flux Ratio Map}

The dust continuum maps taken at two different wavelengths allow us to calculate a flux ratio map. Note that such a ratio depends on two parameters: the emissivity spectral index, $\beta$, and the temperature of the dust grains, $T_{\text {dust }}$.

Figure 3 shows a map of the $350 \mu \mathrm{m} / 450 \mu \mathrm{m}$ flux ratio. Note that the $350 \mu \mathrm{m}$ data have been smoothed to the same angular resolution as the $450 \mu \mathrm{m}$ image. Only data above $2 \sigma$ have been used in the computation of the ratio. The plotted ratio range is between 2 and 3 in order to show the change of the ratio clearly.

The flux ratio map reveals a few intriguing features. First of all, the flux ratio toward the clump center is low $(\sim 2)$ and shows a bipolar structure elongated in the north-south direction. Second, the flux ratio increases toward the outer region and reaches the maximum $(\sim 3)$ at $r \sim 0.3 \mathrm{pc}$. Such a high flux ratio is found in all directions and traces a region elongated in the north-south direction. Third, the flux ratio decreases down to $\lesssim 2$ beyond the high flux ratio region at $r \gtrsim 0.4 \mathrm{pc}$.

This variation of the flux ratio can be found also in Figure 4. Here, the flux ratio is calculated over circular annuli with a $10^{\prime \prime}$ width and a $10^{\prime \prime}$ step. The error bars are the standard deviation of the ratio values measured in each annulus. Note that error of the flux ratio increases toward the clump edge, as the intensities of the continuum emission decrease.

Considering the fact that there is only one prominent heating source, the massive (proto)star, inside the dense clump, the flux ratio pattern is expected to be spherically symmetric. However, the observed pattern of the flux ratio map looks significantly elongated, which may be due to the bipolar outflow from the massive (proto)star. This will be discussed in detail in $\S 4$.

\subsection{Molecular Components Traced with $\mathrm{CO}$ Emission}

We use our maps of the clump in the $\operatorname{CO} J=6-5$ line to investigate the physical properties of the molecular gas $(\S 3.2 .1)$

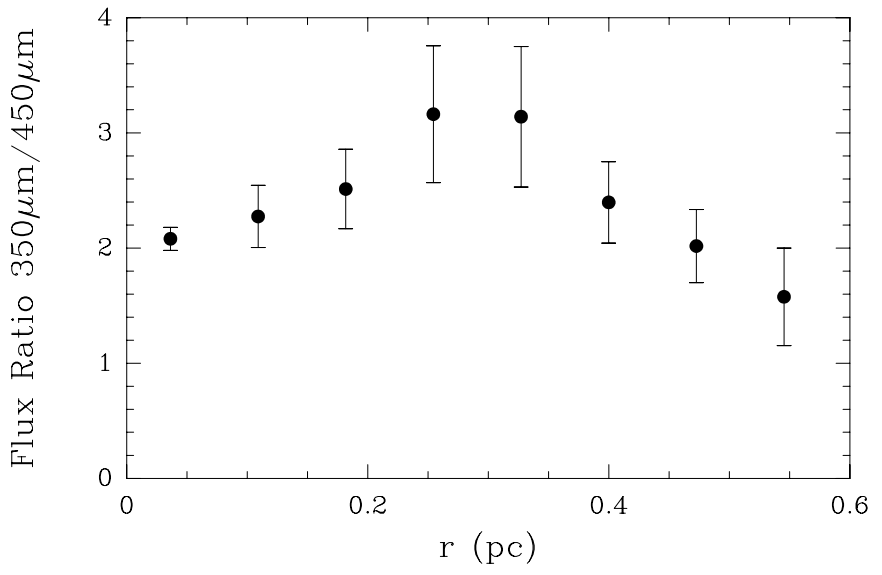

FIG. 4.-Plot of the $350 \mu \mathrm{m} / 450 \mu \mathrm{m}$ flux ratio as a function of the clump radius. The bars are the standard deviation of the ratio in each annulus.

as well as its low-velocity ( $\S 3.2 .2)$ and high-velocity components $(\S 3.2 .3)$.

\subsubsection{Physical Properties Estimated from $\mathrm{CO} J=6-5$ and $J=7-6$ Spectra}

In Figure $5 a$, the $\mathrm{CO} J=7-6$ spectrum (Kawamura et al. 1999 ) is compared with the CO $J=6-5$ spectrum to infer the physical properties of the molecular gas. Figure $5 b$ shows the ratio of the main beam temperatures of these transitions. Since the temperature scale of the CO 7-6 spectrum is a lower limit (Kawamura et al. 1999), the brightness temperature ratio is to be taken as an upper limit. One can see that the redshifted wing has a higher ratio than the blueshifted one.

When dust couples well with molecular gas, the excitation temperature of the gas must be close to the temperature estimated from the spectral energy distribution (SED) of the dust continuum. Therefore line emission is expected to be optically thin when $T_{\mathrm{mb}}$ is lower than $T_{\text {dust }}=T_{\text {ex }}(\sim 40 \mathrm{~K}$; Shepherd et al. 2000 ; see also

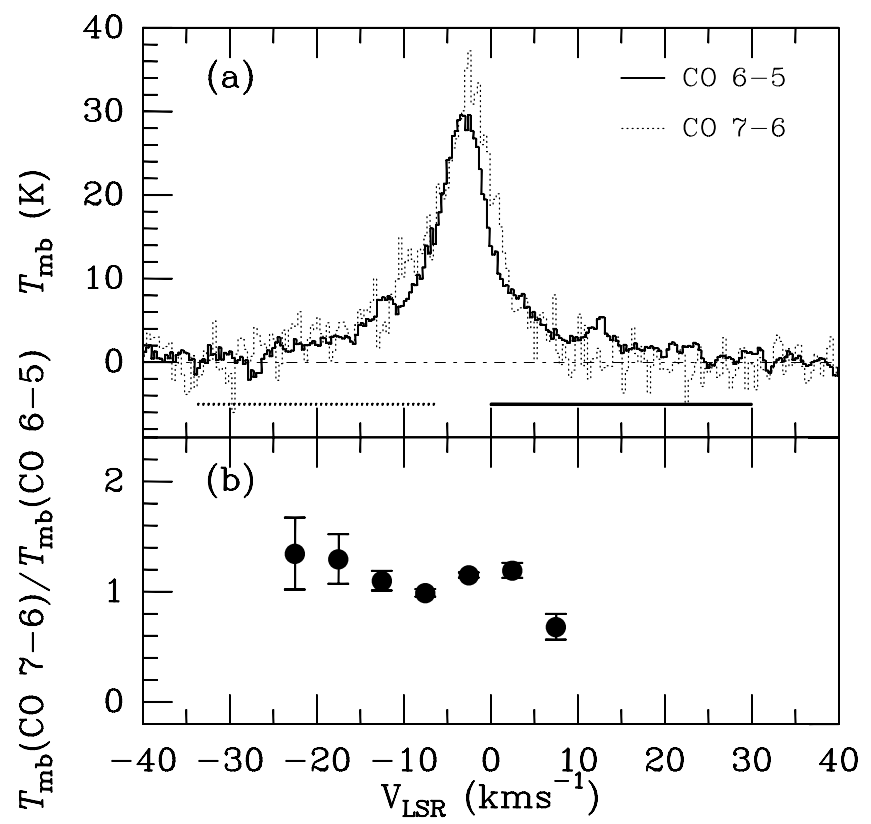

FIG. 5.- (a) CO 6-5 and 7-6 spectra observed toward the center of the clump. The solid and dashed bars under the spectra indicate the ranges of redand blueshifted components (see $\S 3.2 .3$ ). (b) Ratio of the brightness temperatures of the $\mathrm{CO} 7-6$ and 6-5 emission. 
$\S 4.1)$. Thus, the $\mathrm{CO}$ emission in the line wings is likely optically thin, because $T_{\mathrm{mb}} \ll 40 \mathrm{~K}$ at high velocities.

We now wish to obtain an estimate of the gas temperature in the high-velocity gas (i.e., in the line wings) using the results from Figure $5 b$. For this purpose we express the ratio between the main-beam brightness temperatures of the $\mathrm{CO} 7-6$ and 6-5 lines as

$$
\begin{aligned}
\frac{T_{\mathrm{mbCO} 7-6}}{T_{\mathrm{mbCO}-5}}= & \frac{f_{7-6}}{f_{6-5}}\left(\frac{\nu_{6-5}}{\nu_{7-6}}\right)^{2} \frac{B_{\nu_{7-6}}\left(T_{\mathrm{ex}}\right)-B_{\nu_{7-6}}\left(T_{\mathrm{bg}}\right)}{B_{\nu_{6-5}}\left(T_{\mathrm{ex}}\right)-B_{\nu_{6-5}}\left(T_{\mathrm{bg}}\right)} \\
& \times \frac{1-\exp \left(-\tau_{7-6}\right)}{1-\exp \left(-\tau_{6-5}\right)},
\end{aligned}
$$

where $T_{\mathrm{mb}}$ is the main-beam brightness temperature of the $\mathrm{CO}$ transition, $f$ is the corresponding beam filling factor, and $B \nu_{J-(J-1)}(T)=\left(2 h \nu^{3} / c^{2}\right)\left[\exp \left(h \nu_{J-(J-1)} / k T\right)-1\right]^{-1}$ is the Planck function. Here $h$ and $k$ are the Planck and Boltzmann's constants. $T_{\mathrm{bg}}$ is the cosmic microwave background temperature $(2.7 \mathrm{~K})$. At the frequencies of the CO 7-6 and 6-5 transitions $\left(\nu_{7-6}=\right.$ $\left.806.652 \mathrm{GHz} ; \nu_{6-5}=691.473 \mathrm{GHz}\right), B_{\nu_{6-5}}\left(T_{\mathrm{bg}}\right)$ and $B_{\nu_{7-6}}\left(T_{\mathrm{bg}}\right)$ are negligible. As already pointed out, in the line wings $\tau \ll 1$, so that $[1-\exp (-\tau)] \simeq \tau$. Since the source is resolved, we also assume $f_{7-6} / f_{6-5} \simeq 1$. Under these approximations, the above equation can be rewritten as

$$
\frac{T_{\mathrm{mbCO} 7-6}}{T_{\mathrm{mbCO6}-5}} \simeq \frac{\nu_{7-6}}{\nu_{6-5}} \frac{\exp \left(h \nu_{6-5} / k T_{\mathrm{ex}}\right)-1}{\exp \left(h \nu_{7-6} / k T_{\mathrm{ex}}\right)-1} \frac{\tau_{7-6}}{\tau_{6-5}} .
$$

Under local thermal equilibrium (LTE) condition, the ratio of the optical depths of the two transitions can be calculated from the following equation

$$
\frac{\tau_{7-6}}{\tau_{6-5}} \simeq \frac{\nu_{7-6}}{\nu_{6-5}} \exp \left(-\frac{h \nu_{7-6}}{k T_{\mathrm{ex}}}\right) \frac{\exp \left(h \nu_{7-6} / k T_{\mathrm{ex}}\right)-1}{\exp \left(h \nu_{6-5} / k T_{\mathrm{ex}}\right)-1} .
$$

Substituting equation (3) into equation (2), replacing $h \nu_{7-6} / k=$ $38.714 \mathrm{~K}$ and $\nu_{7-6} / \nu_{6-5}=7 / 6$, and solving with respect to $T_{\text {ex }}$, one finds

$$
T_{\mathrm{ex}}=\frac{38.714}{\ln \left[(7 / 6)^{2}\left(T_{\mathrm{mbCO6-5}} / T_{\mathrm{mbCO} 7-6}\right)\right]} .
$$

Based on this equation, the blue- and redshifted components have temperatures equal to 130 and $30 \mathrm{~K}$, respectively, and we thus suggest that the temperature of the blueshifted gas is higher than that of the redshifted gas. Please note that the difference on the ratio of the brightness temperatures of the $\mathrm{CO}$ lines could be partly due to difference in densities in the two outflow lobes.

We also attempted an estimate of the optical depth of these transitions using the large velocity gradient (LVG) model. Assuming an $\mathrm{H}_{2}$ volume density of $10^{5}-10^{6} \mathrm{~cm}^{-3}$, a CO column density of $10^{22}-10^{23} \mathrm{~cm}^{-2}$, an abundance ratio between molecular hydrogen and $\mathrm{CO}$ of $10^{4}$ (see also $\S 3.2 .3$ ), and a measured line width of $25 \mathrm{~km} \mathrm{~s}^{-1}$, we obtain $\tau_{7-6}=1.2$ and $\tau_{6-5}=0.7$ from the model. The tau ratio is 0.7 , similar to that obtainable from equation (3).

\subsubsection{Narrow Line-width Rotating Massive Dense Clump}

Figure 6 shows a comparison between the $350 \mu$ m dust continuum and $\mathrm{CO} 6-5$ line emission with local standard of rest (LSR) velocity in the range from -6.75 to $-0.25 \mathrm{~km} \mathrm{~s}^{-1}$. This velocity

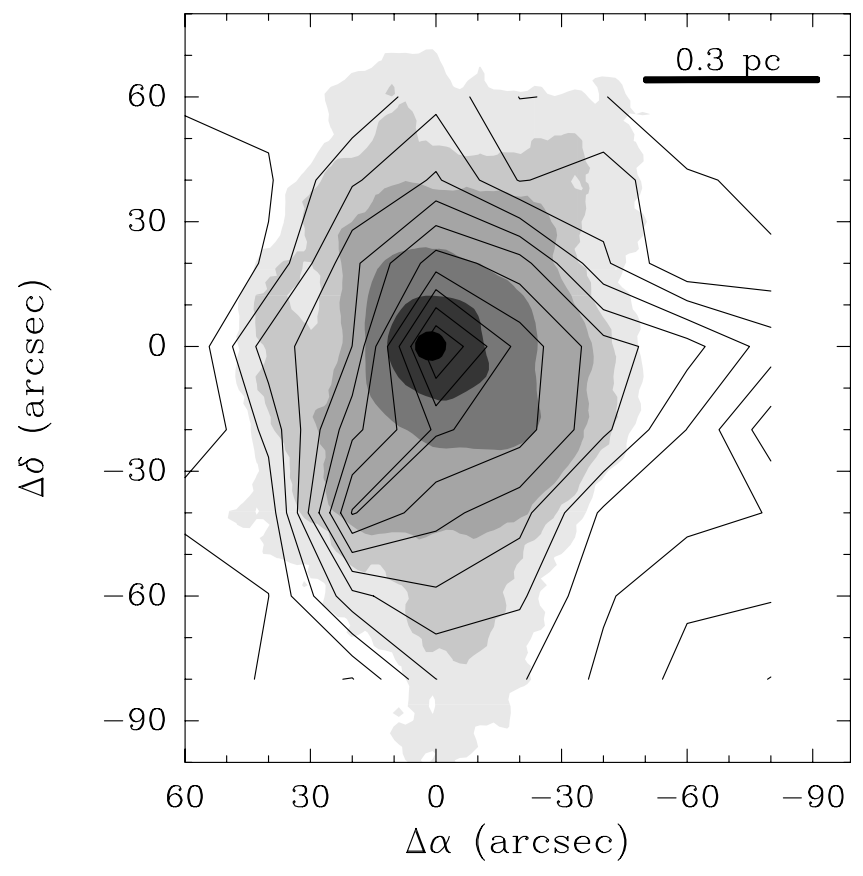

FIG. 6.-Comparison between CO 6-5 line emission close to the systemic velocity (black solid contours) and the $350 \mu \mathrm{m}$ dust continuum emission ( grayscale contours; same as Fig. 1, left). Contours of the CO 6-5 map are drawn in steps of $2 \sigma$, starting from the $2 \sigma$ level $\left(10.6 \mathrm{~K} \mathrm{~km} \mathrm{~s}^{-1}\right)$.

range is chosen so that the emission in the range corresponds to the extended components.

As shown in Figure 6, the warm gas traced by the $\mathrm{CO} 6-5$ line shows good spatial correlation with the $350 \mu \mathrm{m}$ dust continuum emission. The molecular component traced by $\mathrm{CO} 6-5$ is not spherically symmetric. The effective radius of the $\mathrm{CO} 6-5$ emission, $R_{\text {effCO6-5 }}$, is $0.56 \mathrm{pc}\left(77^{\prime \prime}\right)$, larger than $R_{\text {eff } 350}(\S 3.1 .1)$. There is a strong CO 6 -5 peak toward the clump center (i.e., the continuum peak).

The column density of the CO molecule using the $J=6-5$ transition can be derived from (e.g., eq. [A1] of Scoville et al. 1986)

$$
\begin{aligned}
N_{\mathrm{CO} 6-5}= & \frac{3 k}{8 \pi^{3} B_{\mathrm{CO}} \mu_{\mathrm{CO}}^{2}} \frac{e^{E_{\mathrm{CO}_{J}=5} / k T_{\mathrm{ex}}}}{6} \frac{T_{\mathrm{ex}}+\left(h B_{\mathrm{CO}} / 3 k\right)}{1-e^{-h \nu_{\mathrm{C} 06-5} / k T_{\mathrm{ex}}}} \\
& \times \frac{\int T_{\mathrm{mb}} d v}{J_{\mathrm{CO} 6-5}-J_{\mathrm{bg} 6-5}},
\end{aligned}
$$

where $B_{\mathrm{CO}}$ is the rotational constant of the molecule $(57897.5 \mathrm{MHz})$, $\nu_{\mathrm{CO} 6-5}$ the frequency of the transition, $J(T)=\left(h \nu_{\mathrm{CO} 6-5} / k\right) /$ $\left[\exp \left(h \nu_{\mathrm{CO} 6-5} / k T_{\mathrm{ex}}\right)-1\right]$ the specific intensity, and $v$ is the velocity. Note that $T_{\mathrm{mb}}=\left[\tau_{v} /\left(1-e^{-\tau_{v}}\right)\right] T_{b}$, where $T_{b}$ is the brightness temperature. For $\int T_{\mathrm{mb}} d v$, we use an integrated spectrum of the area inside the $2 \sigma$ contour. Since CO 7-6 data are available only for the central region and we do not have isotopic line data, we assume that the column density of molecular hydrogen is proportional to the observed velocity integrated ${ }^{12} \mathrm{CO}$ emission, $\int T_{\mathrm{mb}} d v$.

The LTE mass of the clump, $M_{\mathrm{LTE}}$, is calculated as follows,

$$
M_{\mathrm{LTE}}=A N_{\mathrm{CO} 6-5} \frac{\left[\mathrm{H}_{2}\right]}{[\mathrm{CO}]} \mu_{g} m_{\mathrm{H}_{2}},
$$

with $\left[\mathrm{H}_{2}\right] /[\mathrm{CO}]$ the abundance ratio of molecular hydrogen to $\mathrm{CO}\left(10^{4}\right), \mu_{g}$ the mean atomic weight of the gas (1.36), and $m_{\mathrm{H}_{2}}$ 


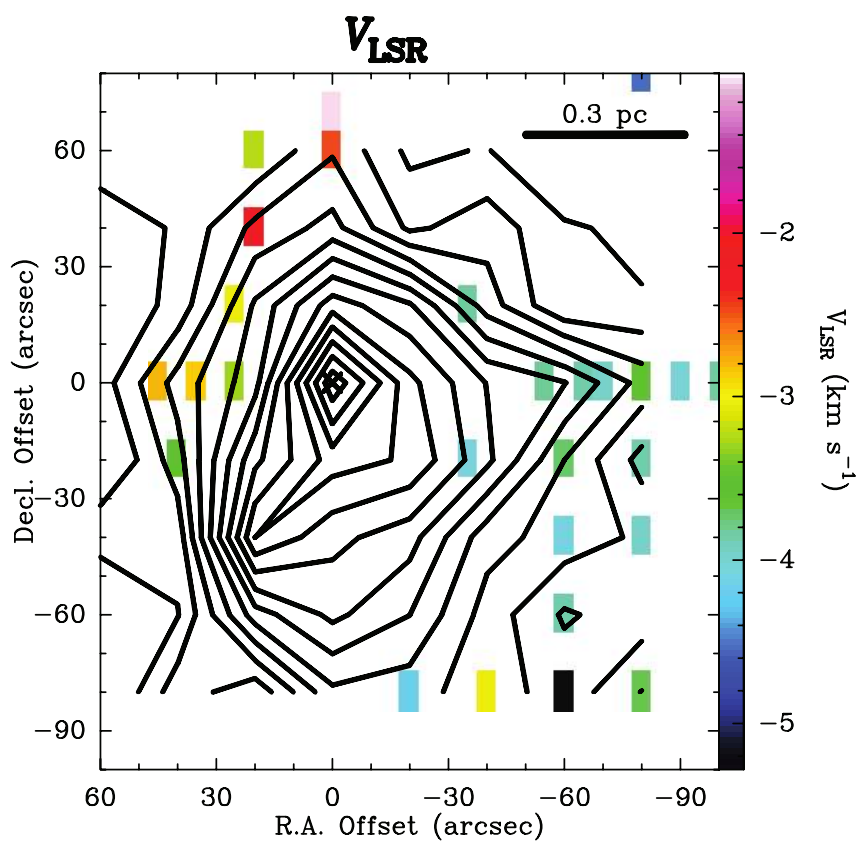

FIG. 7.- Colored pixels in the diagram represent the LSR velocities of the CO 6-5 narrow line width components (contours; same as Fig. 6), overlaid on the map of the CO 6-5 systemic velocity component (contours; same as Fig. 6). The black cross denotes the clump center.

the mass of the hydrogen molecule. The derived LTE mass is $390 M_{\odot}$ for $T_{\mathrm{ex}}=40 \mathrm{~K}$. Hereafter, $40 \mathrm{~K}$ will be used as the temperature of the clump. Please note that the optical depth of CO 6-5 line that traces the extended clump components toward limited central region is likely to be thick. Accordingly, the LTE mass derived above is a lower limit.

Figure 7 shows the LSR velocities toward selected positions where the full width at half-maximum (FWHM) of the line is $\leq 3 \mathrm{~km} \mathrm{~s}^{-1}$. While the east part of the clump has LSR velocities ranging between $\sim-1$ and $-3.5 \mathrm{~km} \mathrm{~s}^{-1}$, in the west side the velocity ranges from -3.0 to $-5.2 \mathrm{~km} \mathrm{~s}^{-1}$. The velocity gradient measured over $1 \mathrm{pc}$ is $\sim 2 \mathrm{~km} \mathrm{~s}^{-1} \mathrm{pc}^{-1}$. The rotation angular velocity, $\Omega$, is $\sim 7 \times 10^{-14} \mathrm{~s}^{-1}$. In Figure 8 we show template spectra of the narrow line width components observed in the east side and west side of the clump. This comparison demonstrates the clear velocity shift from east to west observed across the clump. Closely

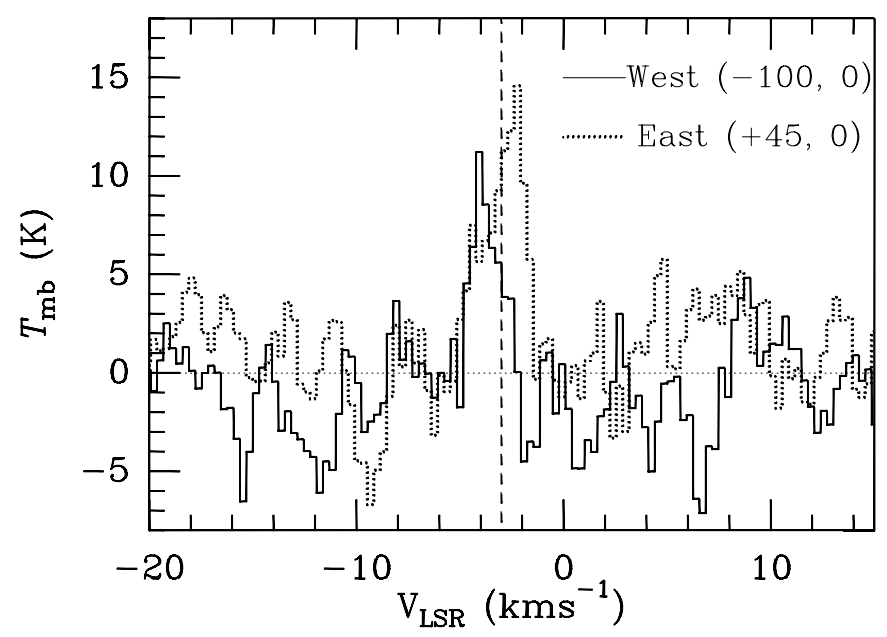

FIG. 8. - The CO $6-5$ spectra observed toward the positions at offsets $\left(+45^{\prime \prime}\right.$, $\left.0^{\prime \prime}\right)$ (east) and $\left(+100^{\prime \prime}, 0^{\prime \prime}\right)$ (west) from the clump center. The dotted vertical line marks $V_{\mathrm{CO} 6-5}$.

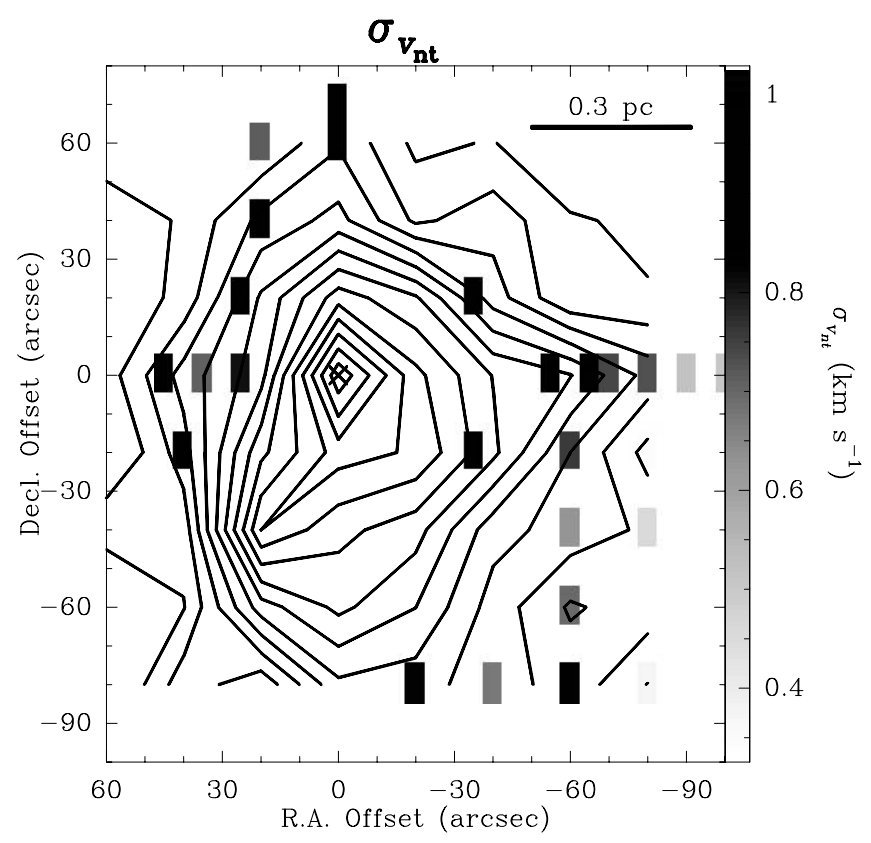

FIG. 9.-Gray scale represents the nonthermal velocity dispersion $\left(\sigma_{v_{\mathrm{nt}}}\right)$ of the narrow line width components, overlaid on the map of CO 6-5 systemic velocity component (contours; same as Fig. 6). The black cross marks the clump center.

looking at the $V_{\text {LSR }}$ change, the velocity shift might be from northeast to southwest. The direction of the velocity gradient is almost perpendicular to the major axis (directed north-south) of the massive dense clump. Assuming that the velocity shift is caused by rotation of the clump and that centrifugal forces balance gravitational ones, one can estimate the equilibrium mass of the molecular dense clump as $M_{\text {rot }} \simeq \Omega^{2} r^{3} / G=200 M_{\odot}$, where $G$ is the gravitational constant. This value agrees reasonably well with the LTE mass, supporting the idea that the velocity gradient may be largely due to rotation of the clump.

We note that rotation of the narrow line-width molecular gas component occurs in the opposite sense with respect to rotation of the circumstellar Keplerian disk associated with the (proto)star. A similar discrepancy in the sense of rotation on different spatial scales has also been observed in W3 (Hayashi et al. 1989) and Orion (Harris et al. 1983), for massive star-forming regions, and in a starless core, L1521F (MC27) in the Taurus molecular cloud (Shinnaga et al. 2004), for low-mass star-forming regions. This suggests that such a complex kinematical structure may not be unusual in the star formation process.

The measured $\Delta v_{\mathrm{FWHM}}$ averaged over the entire $\mathrm{CO}$ 6-5 emitting region is $3.9 \pm 0.2 \mathrm{~km} \mathrm{~s}^{-1}$. From the line width, one can derive the virial mass, $M_{\mathrm{VIR}}$, of the clump,

$$
M_{\mathrm{VIR}} \sim \frac{5+2 e}{3+e} \frac{R_{\mathrm{effCO} 6-5}}{G} \sigma_{v_{\text {total }}}^{2},
$$

where the volume density is proportional to $r^{-e}$. The virial mass is estimated to be $400 M_{\odot}$ for this dense clump. One can assess the equilibrium of the dense clump by comparing this value with the LTE mass and the one obtained assuming centrifugal equilibrium. We note that $M_{\mathrm{LTE}} \simeq M_{\mathrm{rot}}$, and at the same time $M_{\mathrm{LTE}} \simeq$ $M_{\mathrm{VIR}}$. This suggests that the clump is in an equilibrium state and rotation of the core contributes significantly to the equilibrium of the clump.

Figure 9 shows the nonthermal velocity dispersion, $\sigma_{v_{\mathrm{n}}}$, of the narrow line width molecular gas components. Note that the 


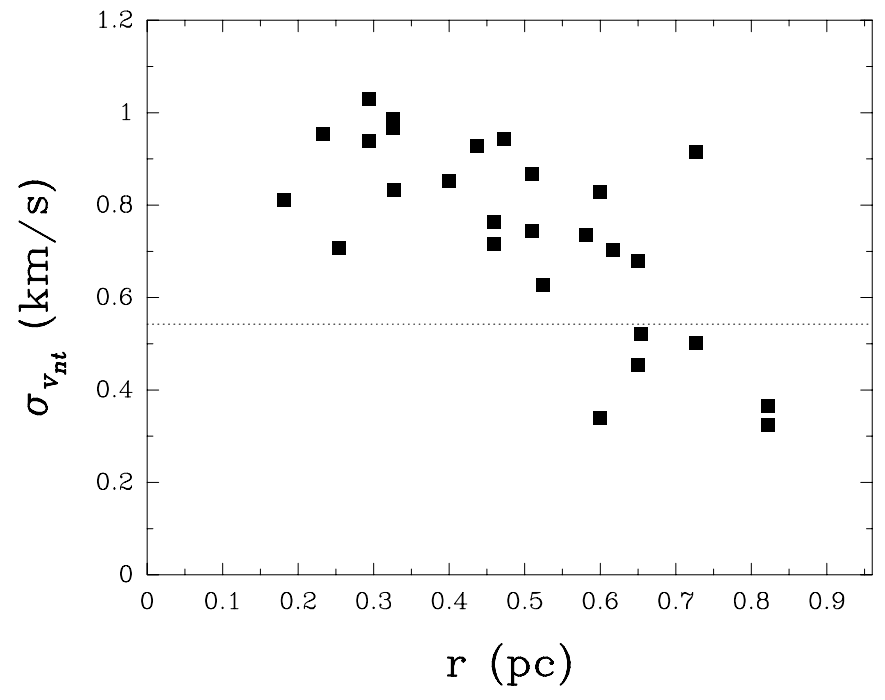

FIG. 10.- Plot of nonthermal velocity dispersion vs. distance from the clump center.

diagram of the velocity width is plotted under an assumption of constant temperature over the core. Since the optical depth of the CO 6-5 line is relatively small (see $\S 3.2 .1$ ), one can estimate the nonthermal velocity dispersion $\left(\sigma_{v_{\mathrm{nt}}}\right)$ by subtracting the thermal line width $\left(k T_{k} / m_{\mathrm{avr}}\right)^{1 / 2}$ from total velocity dispersion, $\sigma_{v_{\text {total }}}=$ $\left[\sigma_{v_{\text {obs }}}^{2}-k T_{k}\left(1 / m_{\mathrm{CO}}-1 / m_{\mathrm{avr}}\right)\right]^{1 / 2}$, where $\sigma_{v_{\mathrm{obs}}}$ is the observed velocity dispersion, $m_{\mathrm{avr}}$ is the average mass unit, and $m_{\mathrm{CO}}=28 \mathrm{amu}$ is the mass of the $\mathrm{CO}$ molecule. $\sigma_{v_{\mathrm{obs}}}$ can be expressed as $\Delta v_{\text {FWHM }}(4 \ln 2)^{-1 / 2}$, where $\Delta v_{\text {FWHM }}$ is the observed line FWHM. From Figure 10, one can see that the nonthermal velocity dispersion decreases with increasing distance from the clump center. To derive the nonthermal velocity dispersion, $40 \mathrm{~K}$ has been used as gas kinetic temperature. The dotted line in the diagram shows the thermal velocity dispersion when $T_{k}=40 \mathrm{~K}$. One can see that the thermal velocity dispersion becomes dominant at $r=0.6 \mathrm{pc}\left(\sim 80^{\prime \prime}\right)$.

\subsubsection{CO 6-5 Large-scale Bipolar Outflow}

Figure 11 shows the CO $J=6-5$ spectra taken toward three positions and the mean spectrum over $\mathrm{a} \leq 120^{\prime \prime}$ area around the clump center. One can see prominent wings in the spectra taken toward each outflow lobe. Blue- and redshifted emission is detected over the velocity ranges $-34.0 \leq V_{\mathrm{LSR}} \mathrm{km} \mathrm{s}^{-1} \leq-6.0$ and $0.0 \leq V_{\mathrm{LSR}} \mathrm{km} \mathrm{s}^{-1} \leq+30$, respectively. The spectrum observed toward the clump center $\left(0^{\prime \prime}, 0^{\prime \prime}\right)$ and the mean spectrum clearly show both red- and blueshifted emission. The spectrum taken toward the center position has a strong peak at $V_{\mathrm{LSR}}=-3 \mathrm{~km} \mathrm{~s}^{-1}$.

Figure 12 shows the distribution of $\operatorname{CO} J=6-5$ blueshifted and redshifted emission superposed on the $350 \mu \mathrm{m}$ dust continuum emission map. Both outflow lobes are located inside the $350 \mu \mathrm{m}$ dust continuum component. The redshifted bipolar outflow lobe is more than twice as extended compared with the blueshifted outflow lobe. Integrated intensities of the blue- and redshifted outflow lobes are measured to be $(9.6 \pm 0.9) \times 10^{2}$ and $\gtrsim(2.5 \pm 0.1) \times 10^{3} \mathrm{~K} \mathrm{~km} \mathrm{~s}^{-1}$, respectively. The masses of the blue- and redshifted outflows are estimated to be 28 and $13 M_{\odot}$, respectively. From the outflow velocity, $\sim 30 \mathrm{~km} \mathrm{~s}^{-1}$, and the extension of the outflow, $\sim 0.6 \mathrm{pc}$, one can estimate the dynamical timescale of the bipolar outflow. The averaged dynamical timescale of the outflow is estimated to be $\gtrsim 1.7 \times 10^{4} \mathrm{yr}$. Note that the estimated age is a lower limit because the south

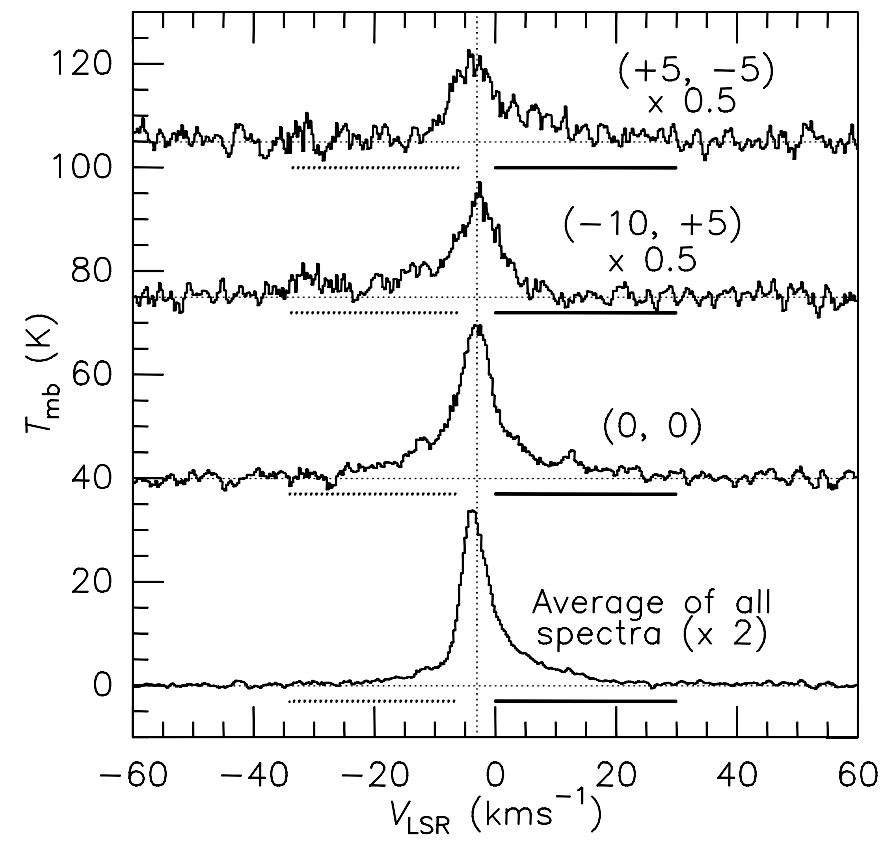

FIG. 11. - The CO $J=6-5$ spectra taken toward three different positions $\left[\left(\right.\right.$ R.A. ${ }_{\text {off set }}^{\prime \prime}$ decl. $\left.{ }_{\text {offset }}\right)=(+5,-5),(-10,+5)$, and $\left.(0,0)\right]$ and average spectrum of all spectra taken within a central area of $1^{\prime} \times 1^{\prime} 20^{\prime \prime}$. The solid and dashed bars under each spectrum show the ranges of red- and blueshifted components. The vertical dashed line is drawn at $V_{\mathrm{CO} 6-5}$.

edge of the redshifted outflow lobe is not fully covered and the inclination angle is unknown. The inclination angle affects the size and velocity. The outflow parameters derived from the $\mathrm{CO}$ 6-5 and 1-0 lines are summarized in Table 3. The outflow velocities obtained from the 6-5 line are higher than those from the $1-0$, while the mean dynamical timescale of the $6-5$ flow is

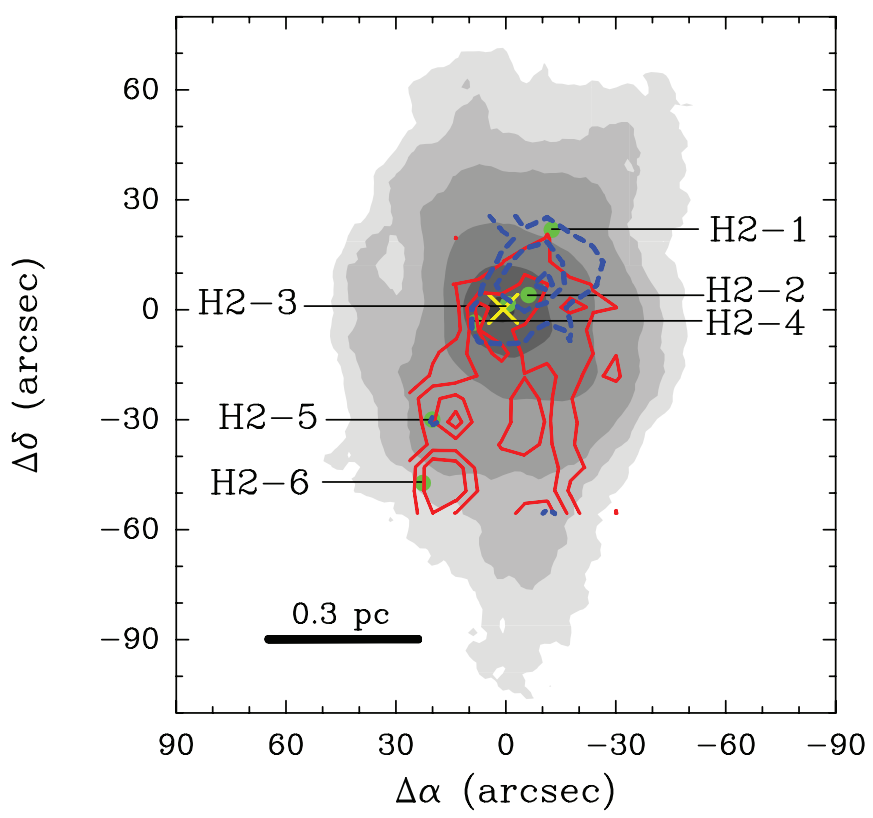

FIG. 12. - The CO $J=6-5$ blueshifted $\left(-34.0 \leq V_{\text {LSR }} \mathrm{km} \mathrm{s}^{-1} \leq-6.0\right.$; blue dashed contours $)$ and redshifted $\left(0.0 \leq V_{\mathrm{LSR}} \mathrm{km} \mathrm{s}^{-1} \leq+30\right.$; red solid contours) emission superposed on the $350 \mu \mathrm{m}$ image (same as Fig. 1). Contours of the CO $J=6-5$ components are drawn in steps of $3 \sigma$, starting from the $3 \sigma$ level $\left(50 \mathrm{~K} \mathrm{~km} \mathrm{~s}^{-1}\right)$. The yellow cross marks the clump center. The shocked $\mathrm{H}_{2}$ knots (green dots) that are marked and numbered in the diagram are taken from Shepherd et al. (2000). 
TABLE 3

IRAS 20126+4104 CO Outflow Parameters

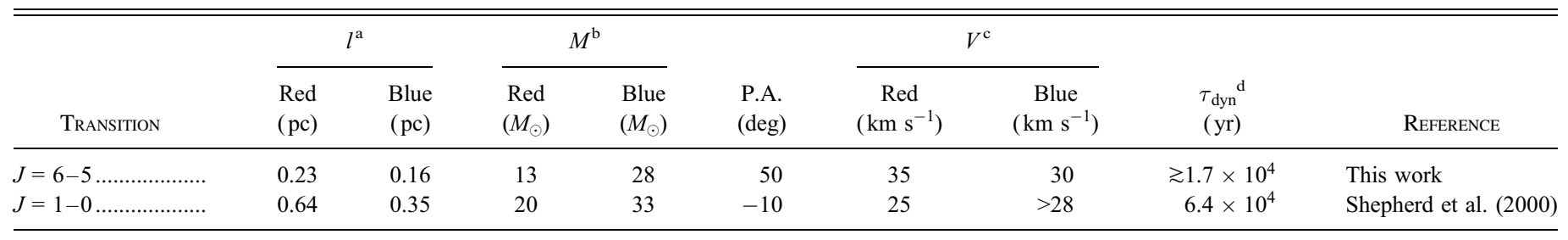

${ }^{\text {a }}$ Outflow lobe radius.

b Mass of the outflow lobes.

c Outer boundary of the wing components.

${ }^{d}$ Dynamical timescale.

shorter than that of the $1-0$. A more detailed discussion of the outflow properties will be given in $\S 4$.

\section{DISCUSSION}

\subsection{Dust Property and Column Density Profile}

To derive the dust mass $M_{\text {dust }}$, we have used the expression (see Hildebrand 1983)

$$
M_{\text {dust }}=\frac{F_{\nu} D^{2}}{B_{\nu}\left(T_{\text {dust }}\right)} \frac{1}{\kappa_{\nu}},
$$

where $F_{\nu}$ is the measured flux density, $D$ the distance to the object, and $B_{\nu}\left(T_{\text {dust }}\right)$ the Planck function.

The coefficient $1 / \kappa_{\nu}$ ( $\kappa_{\nu}$ is dust opacity), is estimated to be $0.1 \mathrm{~g} \mathrm{~cm}^{-2}$ at $250 \mu \mathrm{m}$ in NGC 7023 (Hildebrand 1983). $\kappa_{\nu}$ depends on the frequency as $\kappa_{\nu} \propto \nu^{\beta}$. $\beta$ may range from $\sim 1$ at $\lambda \lesssim$ $200 \mu \mathrm{m}$ to $\gtrsim 2$ at $\sim 1 \mathrm{~mm}$ (Hildebrand 1983 and the references therein). Extrapolating $\kappa_{\nu}$ from $250 \mu \mathrm{m}$ to $350 \mu \mathrm{m}$, one obtains $\kappa_{850 \mathrm{GHz}} \simeq 5.6 \mathrm{~cm}^{2} \mathrm{~g}^{-1} . \beta$ can be estimated from the flux ratio of $350 \mu \mathrm{m}$ and $450 \mu \mathrm{m}$ using the equation

$$
\frac{F_{\nu_{1}}}{F_{\nu_{2}}} \frac{\Omega_{\nu_{1}}}{\Omega_{\nu_{2}}}=\frac{B_{\nu_{2}}\left(T_{\text {dust }}\right)}{B_{\nu_{1}}\left(T_{\text {dust }}\right)}\left(\frac{\nu_{1}}{\nu_{2}}\right)^{\beta},
$$

where $\Omega_{\nu}$ is the beam solid angle. Note that in our case $\Omega_{\nu_{1}} / \Omega_{\nu_{2}} \simeq 1$, because the $350 \mu \mathrm{m}$ image has been smoothed to the same angular resolution as the $450 \mu \mathrm{m}$ image.

Assuming that the clump mass equals $M_{\text {rot }} \simeq 200 M_{\odot}$, one may use equation (8) to derive an estimate of the mean clump temperature, $T_{\text {dust }}=40 \mathrm{~K}$. Then equation (9) gives $\beta \simeq 1.7$. This value lies within the typical range $1.5 \lesssim \beta \lesssim 2.4$ for high-mass starforming regions (e.g., Molinari et al. 2000). The dust temperature is more or less consistent with the temperature, $\sim 44 \mathrm{~K}$, derived from the SED fitting by Shepherd et al. (2000), but lower than the $60 \mathrm{~K}$ estimated by Cesaroni et al. (1999). Note that the errors on $\beta$ and especially $T_{\text {dust }}$ may be significant, as $M_{\text {rot }}\left(200 M_{\odot}\right)$ might not represent the true clump mass. Hofner et al. (2007) estimates a clump mass of $\sim 400 M_{\odot}$ from their SED fitting. The column density toward the clump center is estimated to be $\sim 3.4 \times 10^{23} \mathrm{~cm}^{-2}$, using the values of $\beta$ and $T_{\text {dust }}$ described above. This implies a volume density of $\sim 10^{6} \mathrm{~cm}^{-3}$ toward the clump center. Toward the outflow lobes, the estimated number density becomes $\sim 10^{5} \mathrm{~cm}^{-3}$.

A temperature gradient is likely to exist inside the clump, as this is internally heated by the embedded (proto)star. Such a gradient is likely to be similar to a power law of the type $T(r) \propto$ $r^{-2 /(4+\beta)}$ (Doty \& Leung 1994), with $r$ distance from the clump center. In our case $\beta=1.7$, and hence the power-law index is -0.35 . The effect of this gradient on the estimated density slopes inside the clump is to change the power-law indices of the inner
$(0.13 \lesssim r \lesssim 0.3 \mathrm{pc})$ and outer $(0.3 \lesssim r \lesssim 0.7 \mathrm{pc})$ regions to $\sim-0.9$ and $\sim-2$, respectively. This has been assessed by recalculating the column density profile assuming the clump is spherically symmetric. These indices are not significantly different from those derived without taking the temperature gradient into account. For the nonthermal velocity dispersion described in $\S 3.2 .2$, the velocity dispersion will not change much (order of $0.01 \mathrm{~km} \mathrm{~s}^{-1}$ ), even when we consider an temperature gradient of $T(r) \propto r^{-0.35}$.

An index of -1.26 for the $0.03 \lesssim r \lesssim 0.13$ pc region is only slightly shallower than that typical of a region undergoing free fall $\left[\rho(r) \propto r^{-3 / 2}\right]$, as in the runaway collapse model (LarsonPenston-Hunter solution; Larson 1969; Penston 1969; Hunter 1977 ) and the inside-out collapse model by Shu et al. (1987). Instead, an index of -2.32 for the $0.13 \lesssim r \lesssim 0.3 \mathrm{pc}$ region resembles the density profile $\rho(r) \propto r^{-2}$ of a system in hydrostatic equilibrium. We thus speculate that the inner region of the clump is experiencing infall, whereas the "infalling wave" has not yet reached the outer region beyond $r \gtrsim 0.1 \mathrm{pc}$.

\subsection{Internal Structures of the Dense Clump}

\subsection{1. $\mathrm{CO}$ 6-5 Outflow and Shocked $\mathrm{H}_{2}$ Knots}

From Figure 12, one can see a spatial coincidence between the outflow lobes and the shocked $\mathrm{H}_{2}$ knots. All six knots lie inside the CO $6-5$ bipolar outflow lobes. More specifically, the $\mathrm{CO}$ 6-5 emission shows clear peaks close to the H2-5 and H2-6 knots located in the red shifted lobe. Such a correlation between CO 6-5 peaks and shocked $\mathrm{H}_{2}$ knots indicates that they are physically associated. Note that, unlike the $\mathrm{CO} 6-5$ emission, no peak toward the knot positions is found in the CO 1-0 line, as one can see in Figure 1 of Shepherd et al. (2000). A likely interpretation is that CO 6-5 traces more excited gas compared to the $1-0$ transition. This might be due to the gas at the knot locations being heated and compressed by interaction with the jet powered by the (proto)star.

\subsubsection{Implications from the Submillimeter Dust Radio Map}

There are three major components in the submillimeter flux ratio map (see $\S 3.1 .3$ and Fig. 3), namely (1) a bipolar feature in the central region of the clump, with flux ratio $\simeq 2-2.2$; (2) a region $\sim 0.5 \mathrm{pc}$ in diameter and elongated north-south, with flux ratio starting from $\sim 2-3$ and increasing up to $\gtrsim 3$ close to the outer border; and (3) region of lower flux ratio, $\$ 2$, outside of component (2). Component (1) is located inside component (2). The P.A. of the bipolar structure is about $-17^{\circ}$. Note that features (1) and (2) are detected above a $3 \sigma$ level in the $450 \mu \mathrm{m}$ map and above a $5 \sigma$ level in the $350 \mu \mathrm{m}$ map. Note that $450 \mu \mathrm{m}$ emission in component (3) is detected at a $2-3 \sigma$ level, whereas the $350 \mu \mathrm{m}$ emission is much stronger. Therefore, we believe that all the three features represent real structures. 


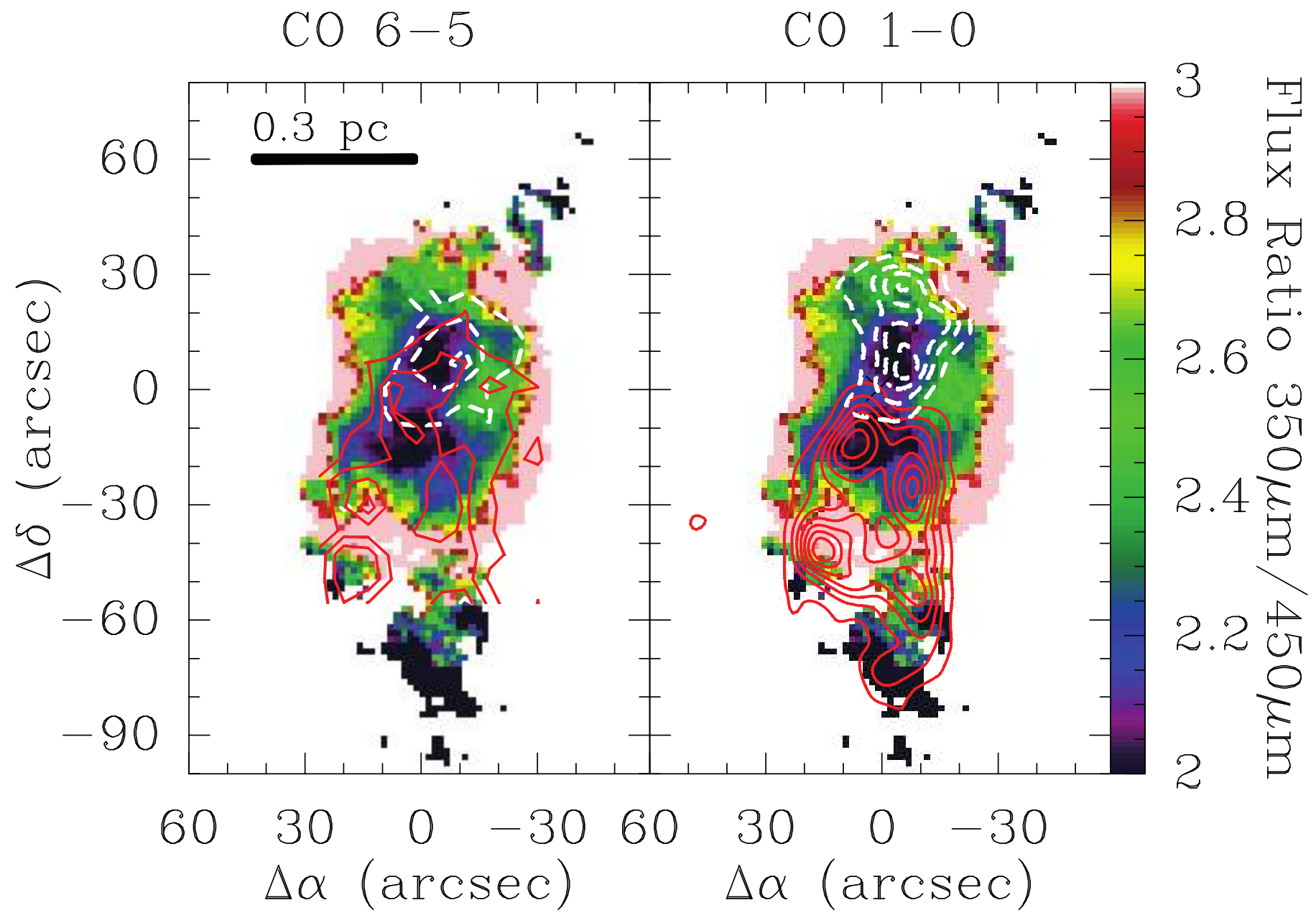

FIG. 13.- The CO 6-5 blue- and redshifted (left; same as Fig. 12), and CO 1-0 blue- and redshifted (right; same as Fig. 1 of Shepherd et al. 2000) maps, superposed on the $350 \mu \mathrm{m} / 450 \mu \mathrm{m}$ flux ratio map. White dashed and red solid contours denote the blue- and redshifted lobes, respectively.

Equation (9) shows that the ratio between the 350 and $450 \mu \mathrm{m}$ fluxes is an increasing function of both $\beta$ and (albeit more weakly) $T_{\text {dust }}$. It is thus impossible to decide whether a change in the ratio is due to a variation of $\beta$, temperature, or both. For example, for $T=40 \mathrm{~K}$, a $\beta$ of 1 and 2 implies a flux ratio of 1.9 and 2.4, respectively. Vice versa, for $\beta=1.7$, a temperature of 20,40 , and $60 \mathrm{~K}$ implies a flux ratio of $1.9,2.3$, and 2.4, respectively. From these numbers one can see that, in order to reproduce flux ratios as large $(\sim 3)$ as those measured on the surface of component (2), very likely an increase of both $\beta$ and $T_{\text {dust }}$ is needed. Hence component (2), which encases the bipolar feature, might be a cocoon of dense material with a warm surface. On the other hand, the lower flux ratio value of component (3) indicates that $T_{\text {dust }}$ and/or $\beta$ in the region ahead of the outflow lobes are lower compared to component (2). We speculate that the higher $T_{\text {dust }}$ and $\beta$ observed on the surface of the cocoon represented by component (2) might be due to a weak shock, propagating from the (proto)star and possibly created by the outflow. Considering a sound speed of $\sim 0.8 \mathrm{~km} \mathrm{~s}^{-1}$, the crossing time of the weak shock would be $\$ 3 \times 10^{5}$ yr. Note that this is likely an upper limit to the age of the outflow (and perhaps of the [proto]star), as shock waves may propagate with larger velocities than the sound speed.

How can the low flux ratio in component (1) be explained? The fact that the shape of this region matches very well the outflow lobes suggests a connection between the two. This suggests that the flow might have swept away the smallest grains from that region, thus biasing the grain size toward the largest ones. This phenomenon would have the effect of reducing the slope of the spectral index $\beta$ and hence of the flux ratio.

In conclusion, the flux ratio variations across the clump strongly suggest that the massive dense clump is no longer uniform in terms of temperature as well as dust properties. To construct a model to explain the observed features, one must keep in mind such inhomogeneities.

\subsubsection{Comparison between Submillimeter Flux Ratio Map and Molecular Components}

Here we make a detailed comparison between the flux ratio map and the structure of the dense clump and outflow. Figure 13 shows the CO 6-5 and CO 1-0 outflow lobes superposed on the flux ratio map. We have already discussed the good correlation between the $\mathrm{CO}$ outflow and the bipolar component (1) of the flux ratio map. Now, we note also that the peak of the blue shifted $\mathrm{CO} 6-5$ lobe is located on the west edge of the northern bipolar feature, which may be due to interaction with the jet detected in $\mathrm{SiO}$ and $\mathrm{H}_{2}$ line emission (Cesaroni et al. 1999) and oriented with P.A. $\simeq-60^{\circ}$. This jet might have excited the molecular gas lying on the west side of the northern lobe. Nothing similar is seen to the southwest that corresponds to the $\mathrm{CO} 6-5$ redshifted outflow lobe. There are two distinct $\mathrm{CO} 6-5$ redshifted peaks associated with the shocked $\mathrm{H}_{2}$ knots, $\mathrm{H} 2-5$ and $\mathrm{H} 2-6$, located at $\left(14^{\prime \prime},-30^{\prime \prime}\right)$ and $\left(17^{\prime \prime},-48^{\prime \prime}\right)$, respectively, as described in $\S 4.2 .1$. At the position of the $\mathrm{H} 2-5$ knot, a high value of the flux ratio $(\sim 3)$ is observed, suggesting relatively large values for both the temperature and $\beta$. Corresponding to the $\mathrm{CO} 6-5$ peak associated 
with knot H2-6, the flux ratio is $\lesssim 2.5$. The difference in flux ratio between $\mathrm{H} 2-5$ and $\mathrm{H} 2-6$ may indicate that the temperature and/or $\beta$ at the position of knot H2-6 could be lower than those of $\mathrm{H} 2-5$.

\section{SUMMARY}

An observational study of the IRAS $20126+4104$ clump is presented. The main findings of this study are summarized as follows.

1. The 350 and $450 \mu \mathrm{m}$ dust continuum images revealed that the power-law index of the volume density in the inner part of the clump is $-1.26 \pm 0.03(r \leq 0.1 \mathrm{pc})$, shallower than that in the outer part, $-2.32 \pm 0.02(0.3 \leq r \leq 0.1 \mathrm{pc})$. The power-law indices suggest that the inner region might be infalling, while the outer region of the clump is still in hydrostatic equilibrium.

2. A map of the peak velocity of narrow line width $\mathrm{CO} 6-5$ components reveals a velocity gradient across the clump, suggesting rotation about an axis oriented northwest-southeast. The rotation occurs in the opposite sense with respect to the circumstellar Keplerian disk associated with the massive (proto)star.

3. Assuming centrifugal equilibrium, one estimates an enclosed mass $M_{\text {rot }}$ of $\sim 200 M_{\odot}$. Estimated $M_{\mathrm{LTE}}$ and $M_{\mathrm{VIR}}\left(\sim 400 M_{\odot}\right)$ of the clump are comparable to $M_{\text {rot. }}$. This indicates that the clump is in equilibrium state as a whole and rotation contributes a large fraction to support the clump from gravitational collapse on large clump scale. At a radius of $0.1 \mathrm{pc}$, the rotation may be disconnected from the inner region and the medium inside of $r \sim 0.1 \mathrm{pc}$ may be infalling. This might be partly related to the disagreement of the rotational axes of the large-scale clump and of the disk associated with the massive (proto)star.
4. The mean spectral index $\beta$ and dust temperature are estimated to be 1.7 and $40 \mathrm{~K}$, respectively.

5. The $350 \mu \mathrm{m} / 450 \mu \mathrm{m}$ ratio map outlines three major components, namely (1) a bipolar feature, which may represent the region swept by the bipolar outflow lobes; (2) a dense cocoon $\sim 0.5 \mathrm{pc}$ in diameter, with a warm surface; and (3) a lowtemperature/low- $\beta$-component outside component (2). These three components indicate that the temperature as well as the nature of the dust (grain size distribution and composition) may vary significantly inside the dense clump. The warm layer of component (2) might have been created by a shock propagating from the (proto)star. The crossing time of the shock is estimated to be $\gtrsim 3 \times 10^{5} \mathrm{yr}$.

6. Strong correlation between $\mathrm{CO} 6-5$ peaks and two of the shocked $\mathrm{H}_{2}$ knots are identified, indicating that they are physically associated.

This research was performed at the Caltech Submillimeter Observatory, supported by NSF grant AST-0229008. H. S. is grateful to Hiroshige Yoshida for the observational support in 2004 July and Debra Shepherd and J. H. Kawamura for providing us their data. H. S. is indebted to Peter Schilke for his LVG code. Thanks are also due to Thomas A. Bell, John Carpenter, and Roger Hildebrand for valuable comments and discussions. H. S. also thanks the Gildas development team (http://www.iram .fr/IRAMFR/GILDAS), which provides us their data reduction packages. We thank the Hawaiian people for allowing us to observe from the summit of their sacred mountain, Mauna Kea.

Facilities: CSO

\section{APPENDIX}

\section{DISTANCE TO THE MASSIVE DENSE CLUMP}

IRAS 20126+4104 is located in the northwest side of the Cygnus X region. This region (Piddington \& Minnett 1952; Davies 1957) is one of the most active star-forming complexes in the Galaxy. Here one can find a large variety of objects, including OB associations, atomic diffuse clouds, molecular dense clouds/cores, evolved stars, and supernova remnants. The distance of the massive dense clump under study is not well established, as it lies on the tangential direction of the spiral arm or bridge structure to which the Sun belongs in our Galaxy (Sharpless 1965; Kerr \& Westerhout 1965). Many studies reporting on observations of IRAS 20126+4104 adopt a distance of $1.7 \mathrm{kpc}$, based on the value quoted by Dame \& Thaddeus (1985). However, Dame \& Thaddeus (1985) do not explain how such a distance was derived. The kinematical estimate using a galactic rotation model may not be accurate for this source because of the location of the source relative to the Galactic center. An alternative method is that of looking for a possible interaction of the IRAS $20126+4104$ clump with nearby objects whose distances are known. From the northeast to the north of the source there is a chain of $\mathrm{H}$ II regions whose northern edge coincides with IC 1318a, the biggest H II region in the cluster. IRAS 20126+4104 is located close to the southwestern edge of the chain. The angular distance from the closest $\mathrm{H}$ II region is about $7^{\prime}$. In addition, there are a number of $\mathrm{H}$ II regions to the east of the source and one supernova remnant (SNR), G 78.2+2.1 (Higgs et al. 1977; Landecker et al. 1980) with a diameter of $\sim 62^{\prime \prime}$ to the southeast of it. The angular distance between the dense clump and the supernova remnant is about $1.5^{\circ}$, along a direction with P.A. $\sim 120^{\circ}$. To the southeast of the object, there is an O star, HD 193322 (O9 V). The angular separation between HD 193322 and IRAS $20126+$ 4104 is about $51^{\prime}$. The distance of the $\mathrm{O}$ star is estimated to be $\sim 1 \mathrm{kpc}$ from photometry (Cruz-Gonzalez et al. 1974). To the southwest, there is no strong $\mathrm{H}$ if region. Ionized gas appears to surround the massive dense clump except on the southwest side, as shown in Figure $1(\S 3.1)$, which is evidence of interaction between our clump and all these objects. The ionized gas is likely due to irradiation from these $\mathrm{H}$ II regions and from the SNR. As the distance of these sources is reported to be $1.5 \pm 0.5 \mathrm{kpc}$ (Ikhsanov 1960; Dickel et al. 1969; Higgs et al. 1977; Landecker et al. 1980; Piepenbrink \& Wendker 1988, and references therein), in this paper we assume a distance of $1.5 \mathrm{kpc}$ for IRAS $20126+4104$.

\section{REFERENCES}

Ayala, S., Curiel, S., Raga, A. C., Noriega-Crespo, A., \& Salas, L. 1998, A\&A, 332,1055

Beuther, H., Schilke, P., Menten, K. M., Motte, F., Sridharan, T. K., \& Wyrowski, F. 2002, ApJ, 566, 945

Beuther, H., et al. 2005, ApJ, 632, 355

Cesaroni, R., Felli, M., Jenness, T., Neri, R., Olmi, L., Robberto, M., Testi, L., \& Walmsley, C. M. 1999, A\&A, 345, 949
Cesaroni, R., Felli, M., Testi, L., Walmsley, C. M., \& Olmi, L. 1997, A\&A, 325,725

Cesaroni, R., Neri, R., Olmi, L., Testi, L., Walmsley, C. M., \& Hofner, P. 2005, A\&A, 434, 1039

Cruz-Gonzalez, C., Recillas-Cruz, E., Costero, R., Peimbert, M., \& TorresPeimbeert, S. 1974, RevMexAA, 1, 211

Dame, T. M., \& Thaddeus, P. 1985, ApJ, 297, 751 
Davies, R. D. 1957, MNRAS, 117, 663

Dickel, H. R., Wendker, H. J., \& Bieritz, J. H. 1969, A\&A, 1, 270

Doty, S. D., \& Leung, C. M. 1994, ApJ, 424, 729

Dowell, C. D., et al. 2003, Proc. SPIE, 4855, 73

Greenhill, L. J., Gwinn, C. R., Schwartz, C., Moran, J. M., \& Diamond, P. J. 1998, Nature, 396, 650

Goldsmith, P. F., et al. 1981, ApJ, 243, L79

Harris, A., Townes, C. H., Matsakis, D. N., \& Palmer, P. 1983, ApJ, 265, L63

Hayashi, M., Kobayashi, H., \& Hasegawa, T. 1989, ApJ, 340, 298

Higgs, L. A., Landecker, T. L., \& Roger, R. S. 1977, AJ, 82, 718

Hildebrand, R. H. 1983, QJRAS, 24, 267

Hofner, P., Cesaroni, R., Olmi, L., Rodríguez, L. F., Martí, J., \& Araya, E. 2007, A\&A, 465, 197

Hunter, C. 1977, ApJ, 218, 834

Hunter, T. R., Churchwell, E., Watson, C., Cox, P., Benford, D. J., \& Roelfsema, P. R. 2000, AJ, 119, 2711

Ikhsanov, R. N. 1960, Soviet Astron., 4, 258

Kawamura, J. H., Hunter, T. R., Tong, C.-Y. E., Blundell, R., Zhang, Q., Katz, C. A., Papa, D. C., \& Sridharan, T. K. 1999, PASP, 111, 1088

Kerr, F. J., \& Westerhout, G. 1965, in Galactic Structure, ed. A. Blaauw \& M. Schmidt (Chicago: Univ. Chicago Press)

Kovacs, A. 2006, Ph.D. thesis, Caltech

Landecker, T. L., Roger, R. S., \& Higgs, L. A. 1980, A\&AS, 39, 133

Larson, R. B. 1969, MNRAS, 145, 271
Lebrón, M., Beuther, H., Schilke, P., \& Stanke, T. 2006, A\&A, 448, 1037 Leong, M. M. 2005, URSI Conf. Sec. J3-10 (Ghent: URSI), http://astro .uchicago.edu/ursi-comm-J/ursi2005/rf-telescope-fabrication/leong

Molinari, S., Brand, J., Cesaroni, R., \& Palla, F. 2000, A\&A, 355, 617

Penston, M. V. 1969, MNRAS, 144, 425

Piddington, J. H., \& Minnett, H. C. 1952, Australian J. Sci. Res., A5, 17

Piepenbrink, A., \& Wendker, H. J. 1988, A\&A, 191, 313

Plambeck, R. L., Wright, M. C. H., Welch, W. J., Bieging, J. H., Baud, B., Ho, P. T. P., \& Vogel, S. N. 1982, ApJ, 259, 617

Scoville, N. Z., Sargent, A. I., Sanders, D. B., Claussen, M. J., Masson, C. R., Lo, K. Y., \& Phillips, T. G. 1986, ApJ, 303, 416

Sharpless, S. 1965, in Galactic Structure, ed. A. Blaauw \& M. Schmidt (Chicago: Univ. Chicago Press), 131

Shepherd, D. S., Yu, K. C., Bally, J., \& Testi, L. 2000, ApJ, 535, 833

Shinnaga, H. 2004, Technical Memorandum 2004 Nov. 22 (Pasadena: Caltech), http://www.submm.caltech.edu/ hs/memo/shinnaga-memo04nov.pdf

Shinnaga, H., Ohashi, N., Lee, S.-W., \& Moriarty-Schieven, G. H. 2004, ApJ, 601,962

Shu, F. H., Adams, F. C., \& Lizano, S. 1987, ARA\&A, 25, 23

Sridharan, T. K., Williams, S. J., \& Fuller, G. A. 2005, ApJ, 631, L73

Voellmer, G. M., et al. 2003, Proc. SPIE, 4855, 63

Williams, S. J., Fuller, G. A., \& Sridharan, T. K. 2004, A\&A, 417, 115

Zhang, Q., Hunter, T. R., \& Sridharan, T. K. 1998, ApJ, 505, L151

Zhang, Q., Hunter, T. R., Sridharan, T. K., \& Cesaroni, R. 1999, ApJ, 527, L117 